\title{
A Vegetation Selection Model for Ecological Bank Protection Works based on Improved TOPSIS Method and Its Application
}

\author{
Wei $\mathrm{Xu}^{*}$, Zengchuan Dong, Jie Ren, Jianting Wang, Yue Zhou and Kedi Ma \\ College of Hydrology and Water Resources, Hohai University, Nanjing, China
}

\begin{abstract}
To solve the complexity and difficulty of vegetation species selection for ecological bank protection works in a certain river effectively, an improved technique for order preference by similarity to ideal solution (TOPSIS) that using the multiple attribute decision making is proposed in river control planning. Besides precise number, interval number and triangle fuzzy number and their processing methods are introduced in TOPSIS. Through the improved TOPSIS, the order preference of the vegetation species could be recommended. The case study in Nenjiang Midstream shows that the proposed model is capable of selecting vegetation species for ecological bank protection works and could provide useful information for the regional integrated planning and management of river control.
\end{abstract}

\section{Introduction}

Most of the bank protection works along the river are often made of mortar or dry block stone and concrete. And concrete revetment has now been verified to lead to the degradation of ecology and environment, causing the continuity of hydrology destroyed and biodiversity loss. In recent years, ecological bank protection has become a sort of popular bank protection technique [1]. And a lot of studies have been carried out for analyzing the mechanism of plant bank protection technology [2]. First, when the flood passes through the vegetation fields, the flow velocity is greatly slowed down and the erosion of the soil surface by the flood flow is reduced due to the drag force of the plants. The effect of the waves on the bank are also reduced by the branches of the vegetation. Second, the developed root system can increase the mechanical strength of erosion resistance of soil. Then the collapse and erosion of the river bank is reduced. Third, the growth of roots, stems and leaves can improve the soil structure, increase the soil organic content and enhance the soil moisture content, thereby enhancing soil erosion resistance. Fourth, plants can also purify water quality. They can absorb pollutants such as organic matter, nitrogen and phosphorus in water.

Currently the ecological bank protection is more and more favored by stakeholders decision makers for its advantage in wave and energy dissipation, increasing the stability of the embankment, less investment and its fine harmonization with surroundings. Vetiveria zizanioides was planted on the bench land at the water side of the Jinjiang River embankment in Changsha City for its good bank protection effect and low cost [3]. Reed was adopted and planted in regulation project of BeijingHangzhou Canal Huaian-Huaiyin Ship Lock Section.
The results showed that the reed planting had the best effect of wave absorption [4]. Calamus and narrow leaf bulrush were used to clean the Qinhuai River water, and both of the plants can clean the polluted water in deep autumn [5]. However, due to the characteristics of regional climatic and soil of the special planting area, growth adaption of the different vegetation species and their protection effect, how to select the optimal vegetation species for a given channel is a complex semi-quantificational systemic decision problem.

Nowadays building mathematical models has become an important approach to solve the optimum selection problems. And technique for order preference by similarity to ideal solution (TOPSIS) is a multi-objective decision making approach more commonly applied in the fields of hospital or university ranking [6], groundwater resources carrying capacity evaluation [7] and wetland ecosystem health evaluation [8]. And the greatest advantage is that this approach can make full use of data. The degree of suitability of the vegetation species for ecological bank protection works and its selection is a multi-objective concept, so the use of TOPSIS is feasible in practice.

In this paper, we study vegetation species suitability for ecological bank protection works, and construct an improved technique for order preference by similarity to ideal solution (TOPSIS) based on multiple attributes that include the conditions of precise number, interval number and triangle fuzzy number. The structure is organized as follows: Section 2 presents the classical TOPSIS and describes its deficiencies. Section 3 builds the vegetation species suitability index system, and describes the improved multiple attribute decision making. The interval number, triangle fuzzy number and their processing methods are introduced. Section 4 
performs case study of vegetation species selection in Nenjiang Midstream. Finally, Section 5 concludes the paper.

\section{Classical TOPSIS}

TOPSIS is a very practical technique for dealing with multi-objective decision making problems. In TOPSIS, the concept that is relatively close to measurement is adopted. And steps of the classical TOPSIS are as follows [8]:

(1) Suppose there are $m$ schemes to be selected, and each scheme has $n$ assessment objectives, then the data matrix of the problem is:

$$
X^{\prime}=\left(\begin{array}{cccc}
x_{11}^{\prime} & x_{12}{ }^{\prime} & \cdots & x_{1 n}{ }^{\prime} \\
x_{21}^{\prime} & x_{22}{ }^{\prime} & \cdots & x_{2 n}{ }^{\prime} \\
\vdots & \vdots & \vdots & \vdots \\
x_{m 1}^{\prime} & x_{m 2}{ }^{\prime} & \cdots & x_{m n}{ }^{\prime}
\end{array}\right)
$$

(2) Conduct normalization processing to the above matrix:

$$
r_{i j}=\frac{x_{i j}^{\prime}}{\sqrt{\sum_{i=1}^{m} x_{i j}{ }^{\prime 2}}}
$$

Then the normalized matrix is obtained:

$$
R=\left(\begin{array}{cccc}
r_{11} & r_{12} & \cdots & r_{1 n} \\
r_{21} & r_{22} & \cdots & r_{2 n} \\
\vdots & \vdots & \vdots & \vdots \\
r_{m 1} & r_{m 2} & \cdots & r_{m n}
\end{array}\right)
$$

(3) Determine the weights of all objectives, then the weight vector can be got as follows:

$$
W=\left(w_{1}, w_{2}, \cdots, w_{n}\right)
$$

(4) Multiply weight by normalized matrix to get the weighted decision matrix.

$$
z_{i j}=w_{j} \bullet r_{i j}
$$

(5) Seek for ideal solution $A^{*}$ and negative ideal solution $A^{-}$of the problem. For the goals, the greater the better:

$$
\begin{aligned}
& A^{*}=\max _{i} z_{i j}=\left[a_{1}^{*}, a_{2}^{*}, \cdots, a_{n}^{*}\right] \\
& A^{-}=\min _{i} z_{i j}=\left[a_{1}^{-}, a_{2}^{-}, \cdots, a_{n}^{-}\right]
\end{aligned}
$$

For goals the smaller the better:

$$
\begin{aligned}
& A^{*}=\min _{i} z_{i j}=\left[a_{1}^{*}, a_{2}^{*}, \cdots, a_{n}^{*}\right] \\
& A^{-}=\max _{i} z_{i j}=\left[a_{1}^{-}, a_{2}^{-}, \cdots, a_{n}^{-}\right]
\end{aligned}
$$

(6) Calculate the Euclidean distance from all schemes to the ideal solution and Euclidean distance to the negative ideal solution:

$$
\begin{gathered}
d_{i}^{*}=\sqrt{\sum_{j=1}^{n}\left(z_{i j}-a_{j}^{*}\right)^{2}} \\
d_{i}^{-}=\sqrt{\sum_{j=1}^{n}\left(z_{i j}-a_{j}^{-}\right)^{2}}
\end{gathered}
$$

Where $a_{j}^{*}$ and $a_{j}^{-}$are the elements of ideal solution $A^{*}$ and negative ideal solution $A^{-}$respectively.

(7) Calculate the approach degree $C_{i}$ between schemes and ideal solutions:

$$
C_{i}^{*}=\frac{d_{i}^{-}}{d_{i}^{-}+d_{i}^{*}}
$$

The approach degree is 1 if problem is the same with the ideal solution, and the approach degree is 0 if the problem is the same with the negative ideal solution. Consequently, the closer the approach degree is to 1 , the better the scheme will be. Rank $C_{i}^{*}$ from the largest to the smallest. Then the scheme with the largest approach degree is the best choice.

From the computational steps of conventional TOPSIS above, we can find that the method mainly focuses on the element that is a precise number. But in many practical problems, interval number and triangle fuzzy number are also frequently involved. Therefore, there is a deficiency in the traditional TOPSIS. To expand the applications of the method, the interval number, triangle fuzzy number and their processing methods are introduced.

\section{Vegetation selection model for ecological bank protection works based on improved TOPSIS}

To realize vegetation species selection for ecological bank protection works effectively, a vegetation selection model based on the vegetation species suitability index system and the improved TOPSIS was built.

\subsection{Index system and its weight}

Much is involved in a vegetation species suitability index system for ecological bank protection. As a special vegetation planting at the water side of the embankment, developed root system, good flexibility of the branches, vegetation height are important factors that determine the bank protection effect when floods happen. Considering growing environment of plants, strong resistance to diseases and insect pests, regional climatic and soil characteristics are also taken into account. In addition, the vegetation must have some certain economic values and landscape values for the country wants to raise revenue especially for the people in those poor rural areas and construction of beautiful village is now a national strategy. Thus the vegetation species suitability index system was built and given in Table 1 . From Table 1, the index system was classified into 3 
groups: bank protection subsystem, auxiliary function subsystem and growth habits subsystem. Among them, some indexes may not be expressed as a precise number appropriately. For example, vegetation height can be expressed as a interval number and landscape value can be described qualitatively like excellent, good, medium, poor or very poor. And these five-grade method can be converted into a triangle fuzzy number. In this index system, the weights of each index were also given in Table 1. And indexes weights were determined by expert evaluation method [9].

Table 1. Vegetation species suitability index system for ecological bank protection works in Nenjiang midstream.

\begin{tabular}{|c|c|c|}
\hline Subsystems & Items & Weights \\
\hline \multirow{3}{*}{$\begin{array}{c}\text { Bank } \\
\text { protection }\end{array}$} & Developed root systems & 0.19 \\
\cline { 2 - 3 } & Flexibility of the branches & 0.18 \\
\cline { 2 - 3 } & Vegetation height & 0.11 \\
\hline \multirow{2}{*}{$\begin{array}{c}\text { Auxiliary } \\
\text { function }\end{array}$} & Economic value & 0.07 \\
\cline { 2 - 3 } & Landscape value & 0.07 \\
\hline \multirow{3}{*}{$\begin{array}{c}\text { Growth } \\
\text { habits }\end{array}$} & $\begin{array}{c}\text { Kinds of diseases and insect } \\
\text { pests }\end{array}$ & 0.13 \\
\cline { 2 - 3 } & Cold endurance & 0.13 \\
\cline { 2 - 3 } & Soil adaptability & 0.12 \\
\hline
\end{tabular}

\subsection{Improved TOPSIS}

Among the indexes above, the index kinds of diseases and insect pests belongs to the cost type, and the other 7 indexes belong to the benefit type. Before normalization processing, invert the cost type index into the benefit type:

$$
x_{i j}=1 / x_{i j}^{\prime}
$$

For different attributes, there are corresponding normalization processes .

(a) For the index that is a precise number $\left(x_{i j}\right)$, its normalization processes is the same as formula (2).

(b) For the index that is a interval number $\left(\left[x_{i j}^{L}, x_{i j}^{U}\right]\right)$, its normalization processes is as follows:

$$
\begin{aligned}
& r_{i j}^{L}=\frac{x_{i j}^{L}}{\sqrt{\sum_{i=1}^{m} x_{i j}^{U 2}}} \\
& r_{i j}^{U}=\frac{x_{i j}^{U}}{\sqrt{\sum_{i=1}^{m} x_{i j}^{L 2}}}
\end{aligned}
$$

(c) For the indexes that is a triangle fuzzy number ( $\left.\left[x_{i j}^{L}, x_{i j}^{M}, x_{i j}^{U}\right]\right)$, its normalization processes is as follows [10]:

$$
\begin{aligned}
& r_{i j}^{L}=\frac{x_{i j}^{L}}{\sqrt{\sum_{i=1}^{m} x_{i j}^{U 2}}} \\
& r_{i j}^{M}=\frac{x_{i j}^{M}}{\sqrt{\sum_{i=1}^{m} x_{i j}^{M 2}}} \\
& r_{i j}^{U}=\frac{x_{i j}^{U}}{\sqrt{\sum_{i=1}^{m} x_{i j}^{L 2}}}
\end{aligned}
$$

For different attributes, there are also corresponding methods of determining ideal solution $A^{*}$ and negative ideal solution $A^{-}$of the problem.

(a) For the index that is a precise number, see formula (6).

(b) For the index that is a interval number:

$$
\begin{aligned}
& A^{*}=\left[\max z_{i j}^{L}, \max z_{i j}^{U}\right] \\
& A^{-}=\left[\min z_{i j}^{L}, \min z_{i j}^{U}\right]
\end{aligned}
$$

(c) For the index that is a triangle fuzzy number:

$$
\begin{aligned}
& A^{*}=\left[\max z_{i j}^{L}, \max z_{i j}^{M}, \max z_{i j}^{U}\right] \\
& A^{-}=\left[\min z_{i j}^{L}, \min z_{i j}^{M}, \min z_{i j}^{U}\right]
\end{aligned}
$$

Calculate the Euclidean distance from all schemes to the ideal solution and Euclidean distance to the negative ideal solution:

$$
\begin{aligned}
& d_{i}^{*}=\sqrt{\sum_{j=1}^{n} d_{i j}^{* 2}} \\
& d_{i}^{-}=\sqrt{\sum_{j=1}^{n} d_{i j}^{-2}}
\end{aligned}
$$

Where

(a) For the index that is a precise number:

$$
d_{i j}^{*}=z_{i j}-a_{j}^{*} \quad d_{i j}^{-}=z_{i j}-a_{j}^{-}
$$

(b) For the index that is a interval number:

$$
\begin{aligned}
& d_{i j}^{*}=\sqrt{\left(z_{i j}-a_{j}^{L *}\right)^{2}+\left(z_{i j}-a_{j}^{U *}\right)^{2}} \\
& d_{i j}^{-}=\sqrt{\left(z_{i j}-a_{j}^{L-}\right)^{2}+\left(z_{i j}-a_{j}^{U-}\right)^{2}}
\end{aligned}
$$

(c) For the index that is a triangle fuzzy number:

$$
\begin{aligned}
& d_{i j}^{*}=\sqrt{\left(z_{i j}-a_{j}^{L *}\right)^{2}+\left(z_{i j}-a_{j}^{M *}\right)^{2}+\left(z_{i j}-a_{j}^{U *}\right)^{2}} \\
& d_{i j}^{-}=\sqrt{\left(z_{i j}-a_{j}^{L-}\right)^{2}+\left(z_{i j}-a_{j}^{M-}\right)^{2}+\left(z_{i j}-a_{j}^{U-}\right)^{2}}
\end{aligned}
$$

Then the approach degree $C_{i}$ can be calculated by formula (10). 


\section{Its application}

Nenjiang River is the largest tributary of Songhuajiang River in North-eastern China. It originates from Greater Khingan Range, wanders through the southern semiarid region, passes through the western plain, and finally flows into Songhuajiang River. The total length of this river is about $1370 \mathrm{~km}$ and its drainage area is $2.97 \times 10^{8}$ $\mathrm{km}^{2}$. The basin is characterized by a continental monsoon climate. The mean annual precipitation is about $500 \mathrm{~mm}$ and mainly concentrates between June and September, which accounts for $82 \%$ of total precipitation. The mean annual temperature is $3.2^{\circ} \mathrm{C}$, and the extreme minimum temperature in history records is $-39.5^{\circ} \mathrm{C}$. The special climatic conditions determine that Nenjiang is a flood prone river [11]. At present, due to the bad foundation of embankment itself along Nenjiang Midstream, the waves do great damages to embankment in flood season. To reduce the wave disaster to embankment, it seems particularly important to build ecological bank protection works. Considering the cold weather and soil fertility condition, the index values of 8 vegetation species for ecological bank protection works that include Reed (R), Calamus (C), Thalia dealbata (TD), Giant reed (GR), Salix integra (SI), Typha (T) and Scirpus tabernaemontani (ST) were collected and given in Table 2.

Table 2. Index value of different vegetation species

\begin{tabular}{|c|c|c|c|c|c|c|c|}
\hline Items & $\mathrm{R}$ & $\mathrm{C}$ & $\mathrm{TD}$ & $\mathrm{GR}$ & SI & $\mathrm{T}$ & $\mathrm{ST}$ \\
\hline Developed root systems & Excellent & Poor & Very poor & Excellent & Excellent & Good & Excellent \\
\hline Flexibility of the branches & Good & Medium & Good & Good & Excellent & Good & Medium \\
\hline Vegetation height $/ \mathrm{m}$ & {$[1,3]$} & {$[0.9,1.5]$} & {$[1,2.5]$} & {$[2,4]$} & {$[1,3]$} & {$[1.3,2]$} & {$[1,2]$} \\
\hline Economic value & 5 & 2 & 1 & 4 & 2 & 5 & 2 \\
\hline Landscape value & Good & Medium & Excellent & Very poor & Poor & Medium & Poor \\
\hline Kinds of diseases and insect pests & 2 & 8 & 1 & 1 & 3 & 2 & 2 \\
\hline Cold endurance & Excellent & Medium & Poor & Very poor & Excellent & Excellent & Excellent \\
\hline Soil adaptability & Good & Medium & Good & Good & Poor & Good & Very poor \\
\hline
\end{tabular}

Obtain the data matrix of the problem according to the relationship between qualitative description and triangle fuzzy number.

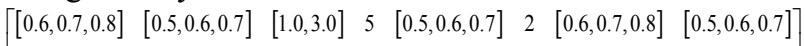
$[0.3,0.4,0.5][0.4,0.5,0.6][0.9,1.5] 2[0.4,0.5,0.6] 8[0.4,0.5,0.6][0.4,0.5,0.6]$ $[0.2,0.3,0.4][0.5,0.6,0.7][1.0,2.5] 1 \quad[0.6,0.7,0.8] 10[0.3,0.4,0.5][0.5,0.6,0.7]$ $[0.6,0.7,0.8][0.5,0.6,0.7][2.0,4.0] 4[0.2,0.3,0.4] 10[0.2,0.3,0.4][0.5,0.6,0.7]$ $[0.6,0.7,0.8][0.6,0.7,0.8][1.0,3.0] 2[0.3,0.4,0.5] 3[0.6,0.7,0.8][0.3,0.4,0.5]$ $[0.5,0.6,0.7][0.5,0.6,0.7][1.3,2.0] 5[0.4,0.5,0.6] 2[0.6,0.7,0.8][0.5,0.6,0.7]$ $[0.6,0.7,0.8][0.4,0.5,0.6][1.0,2.0] 2[0.3,0.4,0.5] 2\left[\begin{array}{lll}2 & 2.6,0.7,0.8] & {[0.2,0.3,0.4]}\end{array}\right]$

Then invert the cost type index kinds of diseases and insect pests into the benefit type by formula (10). Conduct normalization processing to the matrix and multiply weight by normalized matrix to get the weighted decision matrix.

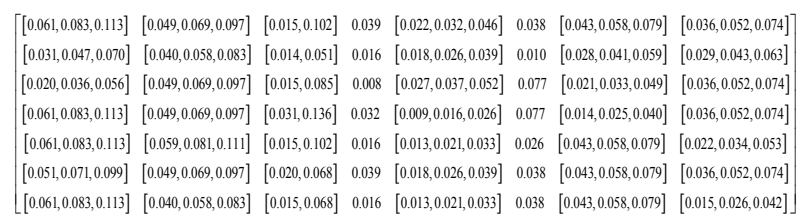

And ideal solution $A^{*}$ and negative ideal solution $A^{-}$of the problem could be obtained.

$$
A^{*}=
$$

$([0.061,0.083,0.113],[0.059,0.081,0.111],[0.031,0.136], 0.039,[0.027,0.037,0.052], 0.077,[0.043,0.058,0.079],[0.036,0.052,0.074])$ $A^{2}=$ $([0.020,0.036,0.056],[0.040,0.058,0.083],[0.014,0.051], 0.008,[0.009,0.016,0.026], 0.010,[0.014,0.025,0.040],[0.015,0.026,0.042])$

Then, the approach degree of 7 vegetation species were calculated by formula (16) and (17) and were shown in Table 3. According to Table 3, we could find that the approach degree of Reed is the largest. It indicates that Reed is the most suitable vegetation species for ecological bank protection works in Nenjiang midstream. Besides, the approach degree of the species Giant reed, Salix integra and Typha are also larger relatively. But among them, Giant reed is not a native species in this region. According to the principle of giving priority to local species, the recommended order of vegetation species for ecological bank protection works in Nenjiang River midstream is the Reed, Salix integra and Typha.

Table 3. The approach degree of different species

\begin{tabular}{|c|c|c|c|}
\hline Species & $d^{*}$ & $d^{-}$ & $C^{*}$ \\
\hline $\mathrm{R}$ & 0.058 & 0.135 & 0.700 \\
\hline $\mathrm{C}$ & 0.140 & 0.052 & 0.270 \\
\hline $\mathrm{TD}$ & 0.115 & 0.099 & 0.463 \\
\hline $\mathrm{GR}$ & 0.073 & 0.149 & 0.670 \\
\hline $\mathrm{SI}$ & 0.080 & 0.125 & 0.610 \\
\hline $\mathrm{T}$ & 0.086 & 0.112 & 0.565 \\
\hline $\mathrm{ST}$ & 0.107 & 0.109 & 0.503 \\
\hline
\end{tabular}

\section{Conclusions}

In this paper, we analyze the deficiencies of conventional TOPSIS and propose an improved method that includes the conditions of precise number, interval number and triangle fuzzy number. Then, we build a vegetation species selection model for ecological bank protection works using the multi-index system. Finally, we validate the effectiveness of the proposed model through the case study in Nenjiang midstream. The model could provide a useful tool for decision-makers in 
aspects such as flood control works planning and ecological remediation.

\section{Acknowledgements}

This work was supported by the Applied Technology Research and Development Program of Heilongjiang Province under Grant No.GZ16B031 and No.GZ16B035 and the Water Resources Department of Shanxi Province under Grant No. 20165011212.

\section{References}

1. D.D. Liu, L. Zhu, J. Zhang, and J. Liang, Select the Optimal Ecological Bank-Protection Technique for Plain-Area Channel Using AHP. Journal of Convergence Information Technology, 2012, 7(1): 214-221.

2. H. Zhao, S.B. Shu, and J.L. Liu, Study on Application of Bank Protection Engineering Technology by Vegetation. Modern Agricultural Science and Technology, 2010, 17:248-250.

3. Q. Wang, Application of Vetiver Grass System in Regulation of Medium and Small Rivers. Hunan Hydro \& Power, 2010, 6:73-75.

4. H.Q. Ge, Y.Q. Zhu, Application of Reed Ecological Bank Revetment in Regulation Project of BeijingHangzhou Canal Huaian-Huaiyin Ship Lock Section. Journal of Nantong University (Natural Science), 2009, 8(2): 62-64.

5. D.W. Yan, "Research on the Adaptability of Submerged Macrophytes Used by Ecological Riverbank in Qinhuai River," Environmental Science and Management, 2006, 31(9):154-156.

6. L. Guo, W.P. Liang, and Y. Han, Comprehensive Evaluation on Medical Quality of a Hospital by TOPSIS Method. Soft Science of Health, 2014, 4:219-221.

7. J.X. Xu, H. Fan, and X.T. Hu, The Model Combined Entropy Weight with Improved TOPSIS and Its Application in Groundwater Resources Carrying Capacity Evaluation. China Rural Water and Hydropower, 2012, 2:30-33.

8. L. Ren, J.T. Liu, J.J. Ni, and X.Y. Xiang, Health Evaluation of a Lake Wetland Ecosystem Based on the TOPSIS Method. Polish Journal of Environmental Studies, 2014, 23(6):2183-2190,.

9. W. Xu, Z.C. Dong, Z.C. Hao, D.Y. Li, and L. Ren, River Health Evaluation Based on the Fuzzy MatterElement Extension Assessment Model. Polish Journal of Environmental Studies, 2017, 26(3):1353-1361.

10. Z.S. Xu, Study on Method for Triangular Fuzzy Number-Based Multi-Attribute Decision Making With Preference Information on Alternatives. System Engineering and Electronics, 2002, 8:9-12.

11. H.Y. Li, M. Xie, and S. Jiang, Recognition Method for Mid- to Long-Term Runoff Forecasting Factors based on Global Sensitivity Analysis in the Nenjiang River Basin. Hydrological Processes, 2012, 26(18):2827-2837. 